\title{
On the Zeros of Tails of Power Series
}

\author{
I. V. Ostrovskii
}

Dedicated to the memory of S. A. Vinogradov

\section{Introduction}

Let

$$
f(z)=\sum_{k=0}^{\infty} a_{k} z^{k}
$$

be a power series with a positive radius of convergence. Let

$$
s_{n}(z)=\sum_{k=0}^{n} a_{k} z^{k}, \quad t_{n}(z)=\sum_{k=n+1}^{\infty} a_{k} z^{k}
$$

be its $n$th section and $n$th tail, respectively.

By now, the distribution of the zeros of $s_{n}(z)$ has been studied in detail; see, e.g., $[2,4,6,7]$, where further references can be found. The distribution of the zeros of the tails $t_{n}(z)$ has been paid less attention. The behavior of the zeros of the tails of some concrete power series was considered in [1, 3, 8, 12-14]. Many important results related to the tails of general power series were obtained in [2] and, especially, in [11]; however, only the moduli of the zeros of tails were treated there.

Some facts related to the arguments of the zeros of tails of power series with infinite radius of covergence were obtained in [10]. These facts show that some restrictions on the arguments of the zeros imply a bound for the growth of the entire function (1.1). Our aim in this paper is to obtain a similar result for power series with a finite radius of convergence. The main result is as follows.

Theorem. Let $f(z)$ be a power series (1.1) convergent in the unit disc D. Suppose there exist two different tails, $t_{m}(z)$ and $t_{n}(z)$, such that all zeros of $t_{m}(z) t_{n}(z)$ lie on a finite system of radii of $\mathbf{D}$. Then

$$
\log M(r, f)=O\left(\frac{1}{(1-r)^{2}}\right), \quad r \rightarrow 1,
$$

where $M(r, f)=\max \{|f(z)|:|z|=r\}$.

Supported in part by INTAS 96-0858 grant. 
The following example shows that the bound (1.2) is the best possible in the sense of order:

$$
f(z)=P(z)+z^{n+1} \cos \frac{z}{(1-z)^{2}}, \quad z \in \mathbf{D}, \quad n \in \mathbf{N},
$$

where $P(z)$ is a polynomial of degree $<n+1$. In this example, the tails

$$
t_{n}(z)=z^{n+1} \cos \frac{z}{(1-z)^{2}}, \quad t_{n+1}(z)=z^{n+1}\left(\cos \frac{z}{(1-z)^{2}}-1\right)
$$

have only real zeros in $\mathbf{D}$.

\section{Proof of the theorem}

Consider the function

$$
q(z)=\frac{t_{n}(z)}{s_{m}(z)-s_{n}(z)}, \quad z \in \mathbf{D}
$$

and observe that

$$
q(z)-1=\frac{t_{m}(z)}{s_{m}(z)-s_{n}(z)} .
$$

Since $s_{m}(z)-s_{n}(z)$ has finitely many zeros, the condition of the theorem implies that all roots of the three equations

$$
q(z)=0, \quad q(z)=\infty, \quad q(z)=1
$$

lie on a finite system of radii of $\mathbf{D}$. Denoting these radii by

$$
\left\{z: \arg z=\alpha_{j}, 0 \leq|z|<1\right\}, \quad 0 \leq \alpha_{1}<\alpha_{2}<\cdots<\alpha_{p}<2 \pi,
$$

we consider the sectors

$$
S_{j}=\left\{z: \alpha_{j}<\arg z<\alpha_{j+1}, 0<|z|<1\right\}, \quad j=1, \ldots, p, \quad \alpha_{p+1}=\alpha_{1}+2 \pi .
$$

In order to investigate the behavior of $q(z)$ and $f(z)$ in $S_{j}$, we need the function

$$
w(\zeta ; \gamma)=\frac{\left(2 \zeta^{\pi / \gamma}-i\right)\left(2-i \zeta^{\pi / \gamma}\right)}{\left(2 \zeta^{\pi / \gamma}+i\right)\left(2+i \zeta^{\pi / \gamma}\right)}, \quad 0<\gamma \leq 2 \pi, 0<\arg \zeta<\gamma
$$

This function conformally maps the sector $\{\zeta: 0<\arg \zeta<\gamma, 0<|\zeta|<1\}$ onto D. A direct calculation shows that

$$
\begin{aligned}
1-\left|w\left(\rho e^{i \theta} ; \gamma\right)\right| & >\left(1-\left|w\left(\rho e^{i \theta} ; \gamma\right)\right|^{2}\right) / 2 \\
& =\frac{12 \rho^{\pi / \gamma}\left(1-\rho^{2 \pi / \gamma}\right) \sin (\pi \theta / \gamma)}{\left(4+\rho^{2 \pi / \gamma}-4 \rho^{\pi / \gamma} \sin (\pi \theta / \gamma)\right)\left(1+4 \rho^{2 \pi / \gamma}+4 \rho^{\pi / \gamma} \sin (\pi \theta / \gamma)\right)} \\
& >(4 / 15) \rho^{\pi / \gamma}\left(1-\rho^{2 \pi / \gamma}\right) \sin (\pi \theta / \gamma) \\
& >C(1-\rho) \sin (\pi \theta / \gamma), \quad 0<\theta<\gamma, 1 / 2 \leq \rho<1,
\end{aligned}
$$

where $C>0$ is a constant independent of $\rho$ and $\theta$. 
The function

$$
w\left(z e^{-i \alpha_{j}} ; \alpha_{j+1}-\alpha_{j}\right), \quad j \in\{1,2, \ldots, p\},
$$

conformally maps $S_{j}$ onto $\mathbf{D}$. Let $z_{j}(w)$ be the function performing the inverse conformal mapping. Then the function

$$
q\left(z_{j}(w)\right), \quad w \in \mathbf{D}
$$

is analytic in $\mathbf{D}$ and does not assume the values 0 and 1 there. By the Schottky theorem (see, e.g., [5, p. 60]), we have the following estimate:

$$
\log ^{+}\left|q\left(z_{j}(w)\right)\right|=O\left(\frac{1}{1-|w|}\right), \quad|w| \rightarrow 1
$$

Since (2.1) implies $f(z)=s_{n}(z)+\left(s_{m}(z)-s_{n}(z)\right) q(z)$, we see that

$$
f\left(z_{j}(w)\right)=s_{n}\left(z_{j}(w)\right)+\left[s_{m}\left(z_{j}(w)\right)-s_{n}\left(z_{j}(w)\right)\right] q\left(z_{j}(w)\right) .
$$

The functions $s_{m}\left(z_{j}(w)\right)$ and $s_{n}\left(z_{j}(w)\right)$ are bounded in $\mathbf{D}$; therefore, (2.4) implies that

$$
\log ^{+}\left|f\left(z_{j}(w)\right)\right|=O\left(\frac{1}{1-|w|}\right), \quad|w| \rightarrow 1
$$

Substituting

$$
w=w\left(z e^{-i \alpha_{j}} ; \alpha_{j+1}-\alpha_{j}\right), \quad z \in S_{j}
$$

we get

$$
\log ^{+}|f(z)| \leq \frac{C_{1}}{1-\left|w\left(z e^{-i \alpha_{j}} ; \alpha_{j+1}-\alpha_{j}\right)\right|}, \quad z \in S_{j}
$$

and, taking (2.3) into account,

$\log ^{+}\left|f\left(r e^{i \varphi}\right)\right| \leq \frac{C_{2}}{(1-r) \sin \left[\pi\left(\varphi-\alpha_{j}\right) /\left(\alpha_{j+1}-\alpha_{j}\right)\right]}, \quad \alpha_{j}<\varphi<\alpha_{j+1}, \frac{1}{2} \leq r<1$,

where the constants $C_{1}, C_{2}>0$ are independent of $z=r e^{i \varphi}$. Evidently, the validity of the latter inequality for $j=1,2, \ldots, p$, implies that

$$
\log ^{+}\left|f\left(r e^{i \varphi}\right)\right| \leq \frac{C}{(1-r) \prod_{j=1}^{p}\left|\varphi-\alpha_{j}\right|}, \quad 0 \leq \varphi<2 \pi, 0<r<1,
$$

where $C>0$ is independent of $r e^{i \varphi}$.

To deduce (1.2) from (2.5), it suffices to apply the following lemma with

$$
h(\zeta)=f\left(\zeta e^{i \alpha_{j}}\right), j=1,2, \ldots, p ; \quad \delta=(1 / 2) \min \left\{\left(\alpha_{j+1}-\alpha_{j}\right): 1 \leq j \leq p\right\} .
$$

Lemma. Let $h(\zeta)$ be a function analytic in the sector

$$
S=\{\zeta:|\arg \zeta| \leq \delta \leq \pi, 0 \leq|\zeta|<1\}
$$

and satisfying the condition

$$
\log ^{+}|h(\zeta)| \leq \frac{B_{1}}{(1-|\zeta|)|\arg \zeta|}, \quad \zeta \in S
$$


where $B_{1}>0$ is a constant independent of $\zeta$. Then

$$
\log ^{+}|h(\zeta)|=O\left(\frac{1}{(1-|\zeta|)^{2}}\right), \quad \zeta \in S,|\zeta| \rightarrow 1 .
$$

This lemma is contained implicitly in the well-known Sjöberg-LevinsonDomar $\log \log$-theorem (see, e.g., $[9$, p. 376]). We present its proof here for the reader's convenience.

Without loss of generality, we can assume that $\delta$ is small, namely, $\sin \delta \leq$ $\sqrt{2} / 4$, because it suffices to prove (2.7) in any sector with smaller $\delta$.

We fix $R, 1 / 2 \leq R \leq 1$, and consider the function

$$
h_{R}(\zeta)=h(\zeta) \exp \left\{-\frac{B_{2}}{(R-\zeta)^{2}}\right\}, \quad \zeta \in S \backslash\{R\}, \quad h_{R}(R):=0,
$$

where $B_{2}>0$ is a constant to be chosen later. This function is analytic in $S \backslash\{R\}$. Moreover, since

$$
\left|h_{R}(\zeta)\right|=|h(\zeta)| \exp \left\{-\frac{B_{2} \cos (2 \arg (R-\zeta))}{|R-\zeta|^{2}}\right\},
$$

it is continuous on

$$
S_{R}:=S \bigcap\left\{\zeta:|\arg (R-\zeta)| \leq \frac{\pi}{8}\right\},
$$

for $R<1$. We show that the constant $B_{2}$ can be chosen independent of $R$ and so large that

$$
\max \left\{\left|h_{R}(\zeta)\right|: \zeta \in S_{R}\right\} \leq B_{3}, \quad \text { for } \quad R<1,
$$

where the constant $B_{3}>0$ is also independent of $R$.

Putting

$$
\begin{gathered}
L_{R}^{(1)}=\partial S_{R} \bigcap\{\zeta:|\arg \zeta|=\delta\}, \quad L_{R}^{(2)}=\partial S_{R} \bigcap\{\zeta:|\arg (R-\zeta)|=\pi / 8\}, \\
M_{R}^{(k)}=\max \left\{\left|h_{R}(\zeta)\right|: \zeta \in L_{R}^{(k)}\right\}, \quad k=1,2,
\end{gathered}
$$

we have

$$
\max \left\{\left|h_{R}(\zeta)\right|: \zeta \in \partial S_{R}\right\}=\max \left\{M_{R}^{(1)}, M_{R}^{(2)}\right\} .
$$

An elementary geometric calculation shows that for $\zeta \in L_{R}^{(1)}$ and $R<1$ we have

$$
|\zeta| \leq\left(R \sin \frac{\pi}{8}\right) / \sin \left(\delta+\frac{\pi}{8}\right)<\left(\sin \frac{\pi}{8}\right) / \sin \left(\delta+\frac{\pi}{8}\right)<1 .
$$

Therefore, $M_{R}^{(1)}$ is bounded by a constant independent of $R$.

Next, (2.6) and (2.8) imply the inequality

$$
M_{R}^{(2)} \leq \max \left\{\exp \left(\frac{B_{1}}{(1-|\zeta|)|\arg \zeta|}-\frac{B_{2} \cos (\pi / 4)}{|R-\zeta|^{2}}\right): \zeta \in L_{R}^{(2)}\right\} .
$$

We set

$$
R-\zeta=t \exp \left( \pm \frac{i \pi}{8}\right), t>0, \quad \text { for } \quad \zeta \in L_{R}^{(2)}, \quad \frac{1}{2} \leq R<1
$$


Elementary geometric calculations show that, for these $\zeta$,

$$
\begin{gathered}
0 \leq t \leq(R \sin \delta) / \sin \frac{\pi}{8}<R \cos \frac{\pi}{8} \\
1-|\zeta|>\left(1-|\zeta|^{2}\right) / 2>\left(R^{2}-|\zeta|^{2}\right) / 2=t\left(2 R \cos \frac{\pi}{8}-t\right) / 2>\left(t R \cos \frac{\pi}{8}\right) / 2>\frac{1}{8} t,
\end{gathered}
$$

and, moreover, there is a positive constant $B_{4}$ independent of $R$ and $B_{4}$ such that

$$
|\arg \zeta| \geq B_{4} t \text {. }
$$

Therefore,

$$
M_{R}^{(2)} \leq \max \left\{\exp \left(\frac{8 B_{1}}{B_{4} t^{2}}-\frac{B_{2} \cos (\pi / 4)}{t^{2}}\right): 0<t<\infty\right\} .
$$

We conclude that if $B_{2} \geq 8 B_{1} /\left(B_{4} \cos (\pi / 4)\right)$, then $M_{R}^{(2)} \leq 1$. Thus, (2.9) is true.

Fix any $\zeta \in \operatorname{int} S_{1}$. Since

$$
\operatorname{int} S_{1} \subset \bigcup_{1 / 2<R<1} S_{R}
$$

letting $R \uparrow 1$ in (2.9), we get

$$
\left|h_{1}(\zeta)\right| \leq B_{3}, \quad \zeta \in \operatorname{int} S_{1} .
$$

Hence, for $\zeta \in \operatorname{int} S_{1}$,

$$
|h(\zeta)|=\left|h_{1}(\zeta) \exp \frac{B_{2}}{(1-\zeta)^{2}}\right| \leq B_{3} \exp \frac{B_{2}}{(1-|\zeta|)^{2}},
$$

i.e., (2.7) is true. For $\zeta \in S \backslash \operatorname{int} S_{1}$ we have $|\arg \zeta| \geq B_{5}(1-|\zeta|)$, so that the validity of (2.7) is an immediate consequence of (2.6).

\section{Some remarks}

$1^{\circ}$. One of the results of $[10]$ mentioned in the Introduction is the following.

Let $f(z)$ be a power series (1.1) with an infinite radius of convergence. Suppose that there exists (i) a finite system of rays

$$
P=\bigcup_{j=1}^{p}\left\{z: \arg z=\alpha_{j}, 0 \leq|z|<\infty\right\}, \quad 0 \leq \alpha_{1}<\alpha_{2}<\cdots<\alpha_{p}<2 \pi
$$

and (ii) three pairwise different tails $t_{m}(z), t_{n}(z), t_{l}(z)$, such that all but finitely many zeros of $t_{m}(z) t_{n}(z) t_{l}(z)$ lie on $P$. Then

$$
\log M(r, f)=O\left(r^{\pi / \gamma} \log r\right), \quad r \uparrow \infty,
$$

where $\gamma=\min \left\{\left(\alpha_{j+1}-\alpha_{j}\right): 1 \leq j \leq p\right\}\left(\alpha_{p+1}=\alpha_{1}+2 \pi\right)$.

This result can be refined by replacing three tails in (ii) by two tails, $t_{m}(z)$ and $t_{n}(z)$, say. For the proof, it suffices to define $q(z)$ by the equation

$$
q(z)=\frac{t_{n}(z)}{s_{m}(z)-s_{n}(z)}, \quad z \in \mathbf{C}
$$


(but not by the equation on the last line on p. 1259 of [10]). The function $q(z)$ defined in this way is meromorphic in $\mathbf{C}$, and all but finitely many roots of the equations (2.2) lie on the rays (3.1). The remaining part of the proof in [10] can be repeated with only trivial changes.

$2^{\circ}$. As mentioned in [10], estimate (3.2) can be refined in the following way by using the Nevanlinna theory for an angle:

$$
\log M(r, f)=O\left(r^{\pi / \gamma}\right), \quad r \uparrow \infty .
$$

This remark remains valid if one replaces three tails by two tails.

$3^{\circ}$. Estimate (1.2) does not depend on the system of radii mentioned in the theorem, meanwhile (3.2) and (3.3) depend on the system of rays (3.1). The example

$$
f(z)=P(z)+z^{n+1} \cos z^{p / 2}, \quad n, p \in \mathbf{N},
$$

where $P(z)$ is a polynomial of degree $<n+1$, and all zeros of $t_{n}(z)$ and $t_{n+1}(z)$ lie on the rays $(3.1)$ with $\alpha_{j}=2(j-1) \pi / p, j=1,2, \ldots, p, \gamma=2 \pi / p$, shows that this dependence is essential and, moreover, (3.3) is sharp in the sense of order.

$4^{\circ}$. The question as to whether $(1.2)$ can be sharpened if there is a finite system of radii such that more than two different tails (e.g., all tails) have all but finitely many zeros situated on that system remains open. A result related to the case of an infinite radius of convergence was obtained in [10].

$5^{\circ}$. If we assume that there exist two different tails, $t_{m}(z)$ and $t_{n}(z)$, that have finitely many zeros in $\mathbf{D}$, then (1.2) can be improved; namely,

$$
\log M(r, f)=O\left(\frac{1}{1-r}\right), \quad r \rightarrow 1 .
$$

The following example (due to A. M. Vishnyakova) shows that the bound (3.4) is also sharp in the sense of order:

$$
f(z)=P(z)+z^{n+1} \exp \frac{2 i z}{1+z^{2}}, \quad z \in \mathbf{D}, \quad n \in \mathbf{N},
$$

where $P(z)$ is a polynomial of degree $<n+1$. In this example, both $t_{m}(z)$ and $t_{n}(z)$ have only one multiple zero at $z=0$ in $\mathbf{D}$.

For the proof of (3.4), we observe that the function $q(z)$ defined by $(2.1)$ admits values 0 and 1 only finitely many times in $\mathbf{D}$ and apply the Schottky theorem in the annulus $\{z: \rho<|z|<1\}$ where $q(z)$ does not admit 0 and 1 at all.

I thank A. I. Il'inskii and A. M. Vishnyakova for their careful reading a preliminary version of this paper and for valuable remarks. 


\section{References}

[1] J. D. Buckholtz, A characterization of the exponential series, Amer. Math. Monthly, 73 (1966), 121-123.

[2] J. D. Buckholtz and J. K. Shaw, Zeros of partial sums and remainders of power series; Trans. Amer. Math. Soc., 166 (1972), 269-284.

[3] J. Dieudonné, Sur les zéros des polynômes-sections de $e^{x}$, Bull. Soc. Math. France, 70 (1935), 333-351.

[4] A. Edrei, E. B. Saff, and R. S. Varga, Zeros of sections of power series, Lecture Notes in Math., 1002 (1983), 1-115.

[5] W. H. J. Fuchs, Topics in the theory of functions of one complex variable, D. Van Nostrand Company, Princeton, N.J., 1967.

[6] T. Ganelius, Sequences of analytic functions and their zeros, Arkiv för Matematik, 3 (1953), 1-50.

[7] T. Ganelius, The zeros of partial sums of power series, Duke Math. J., 30 (1963), $533-540$.

[8] G. W. Hedstrom and J. Korevaar, The zeros of partial sums of certain small entire functions, Duke Math. J., 30 (1963), 519-532.

[9] P. Koosis, The logarithmic integral, I, Cambridge University Press, 1988.

[10] I. V. Ostrovskii, Les séries de puissances dont les restes ont seulement des zéros non-positifs, C. R. Acad. Sci. Paris, Sér. I, 325 (1997), 1257-1262.

[11] M. Pommiez, Sur les restes successifs des séries de Taylor, Ann. Fac. Sci. Univ. Toulouse, (4) 24 (1960), 77-165.

[12] G. Szegö, Über eine Eigenschaft der Exponentialreihe, Sitzungsberichte der Berliner Math. Gesselschaft, 23 (1924), 50-64.

[13] C. Y. Yildirim, A sum over zeros of partial sums of $e^{x}$, J. Ramanujan Math. Soc., 6 (1991), 51-66.

[14] C. Y. Yildirim, On the tails of the exponential series, Canad. Math. Bull., 37 (1994), $278-286$.

Department of Mathematics,

Bilkent University,

06533 Bilkent, Ankara, Turkey,

and

B. Verkin Institute for Low Temperature Physics and Engineering,

310164 Kharkov, Ukraine

E-mail address: iossif@fen.bilkent.edu.tr, ostrovskii@ilt.kharkov.ua 